\title{
Domain wall freezing in KDP-type ferroelectrics
}

\section{Authors: V. Hugo Schmidt, G. Bohannan, D. Arbogast, and G. Tuthill}

NOTICE: this is the author's version of a work that was accepted for publication in Journal of Physics and Chemistry of Solids. Changes resulting from the publishing process, such as peer review, editing, corrections, structural formatting, and other quality control mechanisms may not be reflected in this document. Changes may have been made to this work since it was submitted for publication. A definitive version was subsequently published in Journal of Physics and Chemistry of Solids, [VOL\# 61 (February 2000)] DOI\#10.1016/S0022-3697(99)00294-2

V.H. Schmidt, G. Bohannan, D. Arbogast, and G. Tuthill, "Domain wall freezing in KDP-type ferroelectrics," Journal of Physics and Chemistry of Solids 61, 283-289 (February 2000). http://dx.doi.org/10.1016/S0022-3697(99)00294-2 


\title{
Domain wall freezing in KDP-type ferroelectrics
}

\author{
V.H. Schmidt*, G. Bohannan, D. Arbogast, G. Tuthill \\ Physics Department, Montana State University, Bozeman, MT 59717, USA
}

Received 20 March 1999; accepted 30 July 1999

\begin{abstract}
Hysteresis loops in $\mathrm{KH}_{2} \mathrm{PO}_{4}(\mathrm{KDP})$ and its ferroelectric $(\mathrm{FE})$ isomorphs disappear some $60 \mathrm{~K}$ below $T_{\mathrm{c}}$. This disappearance may result from an order-disorder transition of the domain wall. The lowest energy wall consists of a single layer of nonpolar $\mathrm{H}_{2} \mathrm{PO}_{4}$ groups of Slater energy $\varepsilon_{0}$. Including only the Slater/Takagi interactions predicts that a domain wall can become wider by having small protrusions that then diffuse along the wall. Reducing temperature would decrease domain wall mobility without causing a freezing transition. However, if one includes the Ishibashi dipolar interaction, this dipolar energy is minimized for a zero-entropy smooth domain wall with a particular ordered H-bond arrangement. Accordingly, there could be an order-disorder transition within the wall, if the bias "field favoring this H-bond ordering is not great enough to smear out the transition. We are applying this model to predict domain wall mobility temperature dependence, and simultaneously measuring FE hysteresis in KDP-ferroelectrics to determine the nature and sharpness of this proposed domain wall transition. (C) 1999 Elsevier Science Ltd. All rights reserved.
\end{abstract}

Keywords: A. Inorganic compounds; D. Dielectric properties; D. Ferroelectricity; D. Phase transitions

\section{Introduction}

It is nearly 60 years since the firs observation of a dielectric anomaly deep in the ferroelectric phase of $\mathrm{KH}_{2} \mathrm{PO}_{4}$ (potassium dihydrogen phosphate, or KDP). The crystal structure is shown in Fig. 1 and discussed in Section 3. As the temperature of KDP is reduced to around $60 \mathrm{~K}$ below the transition temperature $(122 \mathrm{~K})$ the coercive fiel suddenly begins to increase and the saturation polarization to decrease (shown in Fig. 2), indicating a rapid fall in domain wall mobility. The origin of this domain wall "freezing" remains a mystery despite numerous attempts at its explanation, and despite the fact that the ferroelectric transition itself (discontinuous at normal pressure, second order at high pressure) has by now been well studied. The purpose of this paper is to suggest, as a mechanism for domain wall freezing, an ordering transition in the wall itself. This ordering may be induced by a local interaction that was originally suggested in order to account for the observed hydrogen arrangements in the antiferroelectric (in compounds such as $\mathrm{NH}_{4} \mathrm{H}_{2} \mathrm{PO}_{4}$ ) as well as ferroelectric phases. At low temperatures, the effect of this interaction is to "flatten the wall, making it energetically unfavorable for individual protons in $\mathrm{O}-\mathrm{H} \cdots \mathrm{O}$ hydrogen bonds to shift position and permit the wall to move.

In this paper we firs review the history of experimental observations, and then describe the statistical mechanical lattice model for KDP used here. In this context of this model, we point out that a Slater-like argument can lead to a prediction of a transition within a domain wall. We then describe qualitative behavior consistent with this picture, which we have observed in Monte Carlo simulations of the model.

\section{Review of past experiments}

The dielectric anomaly in KDP at $\sim 60 \mathrm{~K}$ was firs measured by Busch and Ganz [1] while investigating the prospect of a phase transition below the ferroelectric transition. A hydrogen gas cryostat was used to measure dielectric permittivity and $50 \mathrm{~Hz}$ hysteresis at temperatures down to about $40 \mathrm{~K}$. Hysteresis loops showed nearly constant saturation polarization $P_{\mathrm{s}}$ and coercive fiel $E_{\mathrm{c}}$ from $120 \mathrm{~K}$ down 


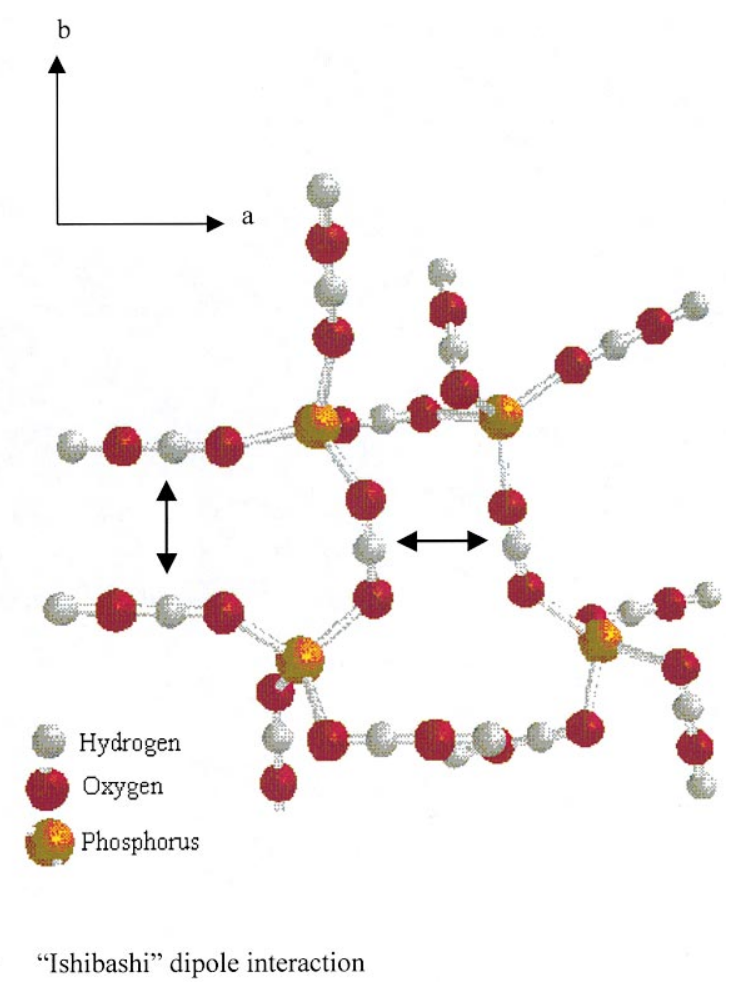

Fig. 1. KDP crystal structure. For simplicity, only one unit cell has been shown and the potassium ions just above each phosphorus (in $+c$ direction) have been suppressed. The arrows indicate the nearest dipole (Ishibashi) interaction.

to about $74 \mathrm{~K}$. Cooling below this temperature caused a steady increase of $E_{\mathrm{c}}$ with an invariant $P_{\mathrm{s}}$ until about $60 \mathrm{~K}$, when the polarizability suddenly diminished. This $60 \mathrm{~K}$ anomaly was ascribed to rapid reduction in domain wall mobility: The speed at which the electric fiel was applied, together with the increasing $E_{\mathrm{c}}$, did not allow enough time for the polarization to switch. Calorimetric measurements [2], demonstrating typical Debye behavior from below $T_{\mathrm{c}}$ to $\sim 16 \mathrm{~K}$, provided further evidence that the anomaly was not a (bulk) structural phase transition.

Electrooptic hysteresis loops [3] measured on DKDP (fully deuterated KDP) demonstrated a similar increase of $E_{\mathrm{c}}$ with the onset at $122 \mathrm{~K}$. Analysis of data comparing $E_{\mathrm{c}}$ to temperature showed a very sharp discontinuity at $\sim 140 \mathrm{~K}$, with a near convergence in $E_{\mathrm{c}}$ to $\sim 2500\left(\mathrm{~V} \mathrm{~cm}^{-1}\right)$ approaching from above. When approaching $140 \mathrm{~K}$ from below, a steep linear dependence of $E_{\mathrm{c}}$ on temperature, starting at $7000\left(\mathrm{~V} \mathrm{~cm}^{-1}\right)$ and decreasing by $-200\left(\mathrm{~V} \mathrm{~cm}^{-1} \mathrm{~K}^{-1}\right)$, was measured.

The anomaly was subsequently investigated by Barkla and Finlayson [4] to examine the reproducibility of the discontinuity at $\sim 60 \mathrm{~K}$ in KDP. Variations from the measurement conditions described by Busch and Ganz included measurement of $P_{\mathrm{s}}$ at dc applied electric field and ac hysteresis loops with applied electric fiel frequency less than $5 \mathrm{mHz}$. The temperature at which the anomaly occurs was shown to be consistent in a number of samples and unaffected by thermal history, demonstrating that the temperature was characteristic of KDP. A domain wall freezing model was developed to explain this behavior.

More recently, the dependence of the freezing temperature $T_{\mathrm{f}}$ (where the maximum in dielectric loss $\varepsilon^{\prime \prime}$ occurs) on experimental conditions has been investigated. Dielectric experiments on KDP by Bornarel [5] indicated that $T_{\mathrm{f}}$ decreases as the ac applied electric fiel amplitude is increased. In similar experiments, the value of $T_{\mathrm{f}}$ has also been shown to decrease with increasing sample thickness along the polar $c$ axis [6], and to increase with higher measurement frequencies [7].

\section{Lattice model}

The structure of KDP is well understood. Each phosphate group is hydrogen-bonded to four other groups in a network equivalent in topology (although not in symmetry) to the diamond lattice. Each bond lies nearly in the $a b$ plane and links two $\mathrm{PO}_{4}$ groups, one situated above the bond (in the $+c$ direction), and the other below the bond. The bond's single proton has two energetically favored positions, one near the bottom of the upper $\mathrm{PO}_{4}$, and the other near the top of the lower group. In the state with full polarization in the $+c$ direction, every proton is positioned near the bottom of a $\mathrm{PO}_{4}$ group; each group then has the two protons below it in 

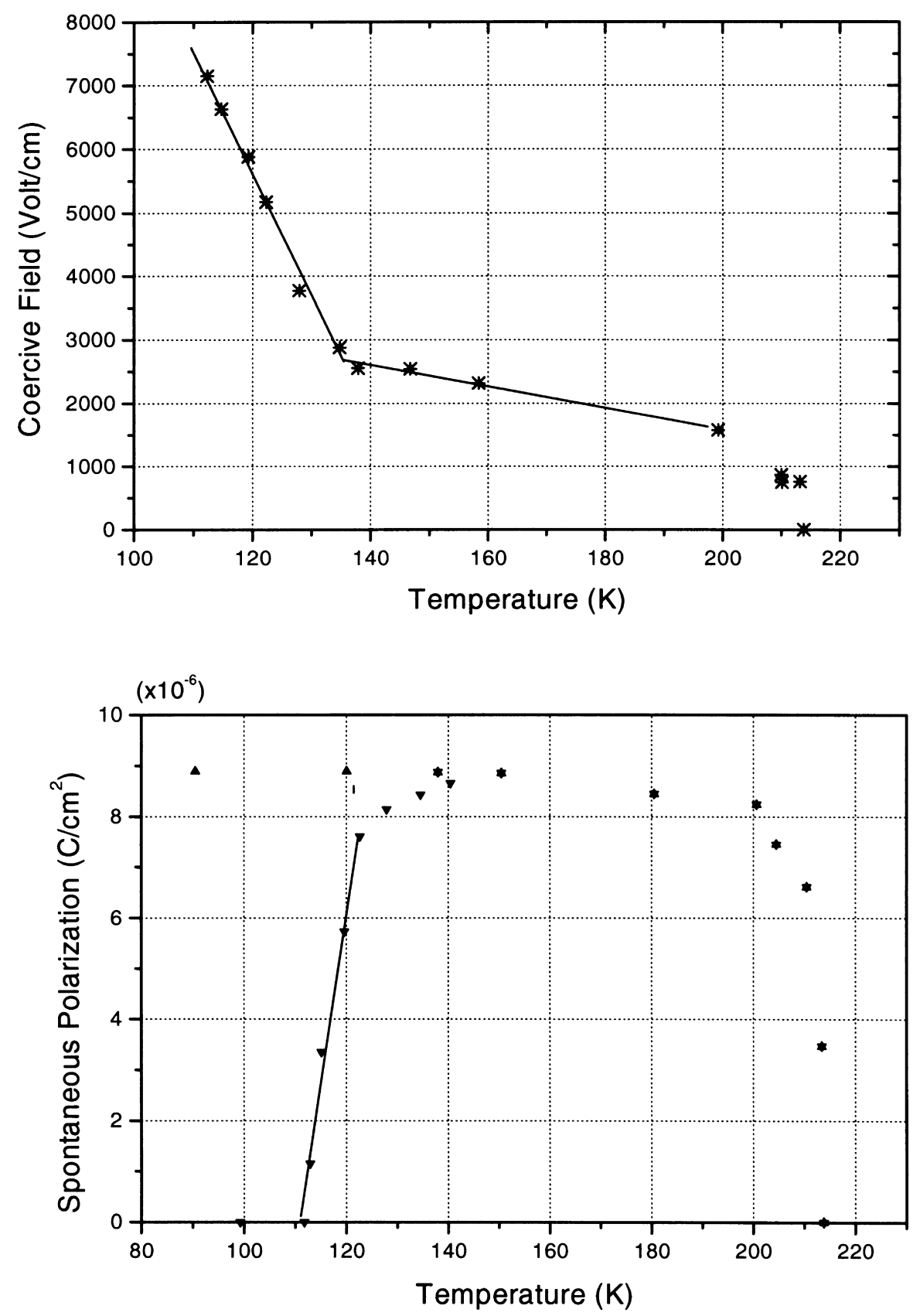

Fig. 2. Spontaneous polarization and coercive fiel versus temperature (from Ref. [3].) Upright and inverted triangles designate total and reversible polarization respectively. Lines serve as a guide to the eye.

nearby positions, and the two protons above it in distant positions. The situation is reversed for the state fully polarized in the $-c$ direction. This ordering of the protons is coupled to the motion of $\mathrm{K}$ and $\mathrm{P}$ along the $c$ axis, accompanied by a shearing of the crystal (the soft mode).

In the "enhanced" Slater-type model used here (originally discussed in Ref. [8]), we assign zero energy to each polar
$\mathrm{PO}_{4}$ group-one whose two adjacent protons are in positions consistent with complete polarization. Other arrangements of the protons have higher energies, and we assign a positive energy to each $\mathrm{PO}_{4}$ in one of the following states:

Slater groups (energy $\varepsilon_{0}$ ): An upper and a lower proton are in nearby positions and the remaining two are in the distant positions. Each of the four such arrangements 
fulfill the "ice rule", of two protons near each $\mathrm{PO}_{4}$ (as do the polar groups). They constitute the groups in observed antiferroelectric analogues of KDP, such as ammonium dihydrogen phosphate.

Takagi groups (energy $\varepsilon_{1} \gg \varepsilon_{0}$ ): Either one or three protons is (are) in the nearby positions. There are eight such configurations

Fully ionized groups (energy $\varepsilon_{2} \gg \varepsilon_{1}$ ): These are the two states in which all protons are nearby, or all are distant.

We do not consider Bjerrum defects, in which a proton is missing from the bond entirely, or conversely two protons are present in or near a single bond. In KDP, these are associated with protonic conduction, which is unobservable at the temperatures of interest here.

Our model also includes interactions, postulated by Ishibashi et al. [8] between protons in parallel bonds staggered along the $c$ axis, assigning energy $\varepsilon_{\mathrm{d}}>0$ to a pair which are located at the same ends of their respective bonds, and energy $-\varepsilon_{\mathrm{d}}$ if the protons are at opposite ends. The arrows in Fig. 1 indicate these interactions. Finally, we also include interactions with an external electric field here always taken to be along the $c$ axis. We assign an energy $\pm p_{\mathrm{s}} E$ to a polar group. Here $p_{\mathrm{s}}=v P_{\mathrm{s}} / 4$, where $v$ is the volume of a crystalline unit cell (containing four phosphate groups) and $P_{\mathrm{s}}$ is the saturation polarization. Both Slater and fully ionized groups are taken to have zero interaction energy with the field whereas Takagi groups have energy $\pm \frac{1}{2} p_{\mathrm{s}} E$.

Two domains, completely polarized in opposite directions along the FE axis, must be separated by a wall containing Slater, Takagi and/or fully ionized groups. Domain walls in KDP are preferentially perpendicular to $a$ or $b$; for specificity we let this wall lie in the $b c$ plane. The lowest energy wall is one consisting of a single layer of Slater groups. If the Ishibashi energy $\varepsilon_{\mathrm{d}}$ is zero, then $\mathrm{PO}_{4}$ groups within the wall are organized in chains of the same Slater type, running along the $b$ direction. Only two of the Slater groups are compatible with this structure, and so the wall entropy is of order $L \ln (2)$, where $L$ is extent of the wall in the $c$ direction. On the other hand, a positive Ishibashi interaction dictates that the lowest energy wall configuratio is unique - that is, the wall consists exclusively of groups all in the same unique Slater configuration

If only Slater groups are allowed in the model (i.e., for $\varepsilon_{1}$, $\varepsilon_{2} \gg \mathrm{k}_{\mathrm{B}} T$ ), a fla wall consisting of a single layer of Slater groups can be widened only if protons connecting loops of at least six $\mathrm{PO}_{4}$ groups simultaneously move to opposite ends of their bonds. This changes two $\mathrm{PO}_{4}$ groups from each domain from polar to Slater $\varepsilon_{0}$ configurations If higher energy groups are allowed (essential for disordering to take place by successive single-proton motions), the domain wall must disorder firs by nucleation of an $\mathrm{HPO}_{4}-\mathrm{H}_{3} \mathrm{PO}_{4}$ pair of Takagi groups within the wall, at an energy cost $2\left(\varepsilon_{1}-\varepsilon_{0}+2 \varepsilon_{\mathrm{d}}\right)$. This pair can then separate as either group moves along the wall in opposite directions along the $c$ axis, converting two polar groups of one sign into Slater groups, and two Slater groups into polar groups of the opposite sign. At zero field the energy cost of one such set of steps (whereby the Takagi group moves out of and then back into the original plane of the wall) in the $\pm c$ direction is $+8 \varepsilon_{\mathrm{d}}$, and results in the net polarization reversal of two $\mathrm{PO}_{4}$ groups. Alternatively, a Takagi pair within the wall can move apart in the $\pm b$ direction, while remaining within the plane of the wall, each step costing energy $4 \varepsilon_{\mathrm{d}}$; this motion itself does not produce a polarization change. In the approximation that wall disordering is created by the separation of such Takagi pairs we may estimate the disordering temperature $T_{\mathrm{w}}$, by using an argument analogous to one which yields the ferroelectric transition temperature in the Slater approximation.

We next briefl review this argument [9] that leads to the Slater transition temperature $T_{\mathrm{S}}$ : Suppose the system is completely ordered ferroelectrically, with the exception of a single pair of Takagi groups connecting a "defect line" of Slater groups. The line may be extended one step by the motion of a Takagi group in one of two directions, and each step costs energy $\varepsilon_{0}$ (assuming $E$ and $\varepsilon_{\mathrm{d}}$ are both zero). So the partition function for this defect line is $1+2 \mathrm{x}+(2 \mathrm{x})^{2}+(2 \mathrm{x})^{3}+\cdots=(1-\mathrm{x})^{-1}$, where $\mathrm{x}=$ $2 \exp \left(-\varepsilon_{0} / \mathrm{k}_{\mathrm{B}} T\right)$. The equilibrium length of the defect line then diverges when $\mathrm{x} \rightarrow 1$, or when $T \rightarrow \varepsilon_{0} / \mathrm{k}_{\mathrm{B}} \ln (2)=T_{\mathrm{S}}$, the Slater transition temperature.

Similarly, the partition function for a defect line between Takagi groups in an otherwise perfect wall is $\left(1-\mathrm{x}^{\prime}\right)^{-1}$, where $\mathrm{x}^{\prime}=\exp \left(-4 \varepsilon_{\mathrm{d}} / \mathrm{k}_{\mathrm{B}} T\right)+\exp \left(-8 \varepsilon_{\mathrm{d}} / \mathrm{k}_{\mathrm{B}} T\right)$. The wall disorders when $\mathrm{x}^{\prime} \rightarrow 1$. Therefore $T_{\mathrm{w}}$ depends on $\varepsilon_{\mathrm{d}}$, and for the observed wall transition temperature of $60 \mathrm{~K}$, we need $\varepsilon_{\mathrm{d}} \approx 7.2 \mathrm{~K}$. We note that when $\varepsilon_{\mathrm{d}}$ is non-zero, the bulk Slater transition temperature must involve both $\varepsilon_{\mathrm{d}}$ and $\varepsilon_{0}$, since the energy cost of lengthening the defect line is modified For a ferroelectric transition temperature of $122 \mathrm{~K}$, the necessary Slater energy, in this approximation, is now given by $\varepsilon_{0} / \mathrm{k}_{\mathrm{B}}=65.7 \mathrm{~K}$.

\section{Monte Carlo simulation}

We have developed a Monte Carlo (MC) simulation of domain wall statics and dynamics in the model described above, using the approach described by Sinitski and Schmidt [10]. The simulated KDP crystal consists of $16 \times$ $16 \times 8$ unit cells, each with four phosphate groups, for a total of $16,384 \mathrm{O}-\mathrm{H} \cdots \mathrm{O}$ bonds. Periodic boundary conditions are applied in the $b$ and $c$ directions, while in the $a$ direction one side of the simulated sample is maintained at positive polarization, and the opposite side at negative polarization. Initially a domain wall is established in a single $b c$ plane in the center of the crystal. Exactly one proton is present in each bond, but all configuration are allowed. Energies and temperature $\mathrm{k}_{\mathrm{B}} T$ are scaled to the Slater energy $\left(\varepsilon_{0} \equiv 1\right)$, and we typically set $\varepsilon_{1}=5, \varepsilon_{2}=15$. The fiel interaction 


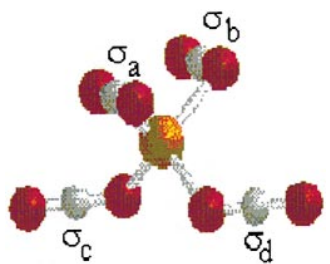

Fig. 3. Phosphate group pseudospin labeling. Note that the two-spin interaction term for upper/upper and lower/lower spins $\left(J_{2}^{\prime \prime}\right)$ is different than for upper/lower pair interactions $\left(J_{2}^{\prime}\right)$, leading to the need for distinction in labeling.

energy is $\varepsilon_{\mathrm{f}}$. The simulation uses a generalized Ising pseudo-spin Hamiltonian with a proton close to the bottom of a phosphate group being assigned spin +1 ; one close to the top of a phosphate group being assigned spin -1 . Bond labeling around a phosphate group is shown in Fig. 3. Spins associated with bonds at the top of a phosphate group are labeled $\sigma_{a}$ and $\sigma_{b}$; spins associated with bonds at the bottom of a phosphate group are labeled $\sigma_{c}$ and $\sigma_{d}$.

The Hamiltonian for a phosphate group is

$$
\begin{gathered}
H(\vec{\sigma})=J_{0}-J_{1} \sum_{i} \sigma_{i}-J_{2}{ }^{\prime} \sum_{\substack{i, j \\
\text { different levels }}} \sigma_{i} \sigma_{j} \\
-J_{2}{ }^{\prime \prime} \sum_{\substack{i, j \\
\text { same level }}} \sigma_{i} \sigma_{j}-J_{4} \sigma_{a} \sigma_{b} \sigma_{c} \sigma_{d} \\
-J_{5} \sum_{\substack{i, j \\
\text { nearest dipole neighbors }}} \sigma_{i} \sigma_{j}
\end{gathered}
$$

With:

$$
\begin{aligned}
& i=a, b, c, d \\
& J_{0}=\frac{1}{4} \varepsilon_{0}+\frac{1}{2} \varepsilon_{1}+\frac{1}{8} \varepsilon_{2} \\
& J_{1}=\frac{1}{2} \varepsilon_{\mathrm{f}} \\
& J_{2}^{\prime}=\frac{1}{8} \varepsilon_{2} \\
& J_{2}^{\prime \prime}=\frac{1}{4} \varepsilon_{0}-\frac{1}{8} \varepsilon_{2} \\
& J_{4}=-\frac{1}{4} \varepsilon_{0}+\frac{1}{2} \varepsilon_{1}-\frac{1}{4} \varepsilon_{2} \\
& J_{5}=\frac{1}{2} \epsilon_{d}
\end{aligned}
$$

The nearest dipole neighbors are as shown by the arrows in Fig. 1.

The simulation uses a standard Metropolis MC scheme with a random sweep of the array. As shown in Table 1, addition of the Ishibashi interaction changes the transition temperature $T_{\mathrm{c}}$. With zero Ishibashi energy, $\mathrm{k}_{\mathrm{B}} T_{\mathrm{c}}$ is approximately 1.4 , while Ishibashi energy of 0.3 produces a shift of $\mathrm{k}_{\mathrm{B}} T_{\mathrm{c}}$ to above 2.1 .

The simulation is designed to probe whether the Ishibashi
Table 1

The addition of the Ishibashi interaction changes the paraelectricferroelectric phase transition temperature $T_{\mathrm{c}}$. The table shows values of $T_{\mathrm{c}} / T_{\mathrm{S}}$ vs $\varepsilon_{\mathrm{d}}\left(\right.$ where $\left.T_{\mathrm{S}}=\varepsilon_{0} / \mathrm{k}_{\mathrm{B}} \ln (2)\right)$, with $T_{\mathrm{c}}$ as derived from simulations

\begin{tabular}{ll}
\hline$\varepsilon_{\mathrm{d}}$ & $T_{\mathrm{c}} / T_{\mathrm{S}}$ \\
\hline 0.0 & $1.4 \pm 0.05$ \\
0.1 & $1.7 \pm 0.1$ \\
0.2 & $1.95 \pm 0.1$ \\
0.3 & $2.1 \pm 0.2$ \\
\hline
\end{tabular}

interaction can account for the texture of the wall during a polarization switching cycle and for the reduction in domain wall mobility at lower temperatures. The condition of fixe opposite polarizations at the boundaries perpendicular to $a$ insures that a wall can be formed without nucleation. This assumes that the crystal has a domain structure in place. One of the outputs of the simulation is an image of an $a b$ plane "slice" of the polarization of a layer one unit cell thick. Each pixel in the image represents the state of a single phosphate group.

Viewing the dynamics of the slice images allows us to discover aspects of switching that traditional hysteresis diagrams alone could not disclose. In particular, we note that including only Slater and Takagi interactions does not produce coherent domain wall motion. Rather, the wall appears to dissolve, while nucleation events within the domain account for the change in net polarization. Moreover, on cooling from a paraelectric state, the domain wall forms but retains fluctuation on varying scales down to temperatures as low as $\frac{1}{2} T_{\mathrm{c}}$. Fig. 4 shows typical domain wall texture as a function of temperature in zero applied field Note that considerable width remains at low temperatures in the $\varepsilon_{\mathrm{d}}=0$ case. The wall width grows considerably under applied fiel for $\varepsilon_{\mathrm{d}}=0$, while the wall remains coherent for $\varepsilon_{\mathrm{d}}=0.3$ in the presence of a field An Ishibashi energy of approximately the same size is needed to produce a fla wall on cooling, and we used this value of 0.3 for hysteresis runs in the ferroelectric regime.

With no Ishibashi interaction, the hysteresis plots indicate switching down to temperatures as low as 0.3. Below this, the activity rates plummet and the simulation bogs down. At all temperatures, polarization switching appears to occur due to nucleation at least as much as due to domain wall motion, as long as the oscillating fiel is large enough to produce saturation. Further investigation is necessary to probe lower frequencies and amplitudes of external fields

With the Ishibashi energy set to 0.3 , the wall remains coherent, with fluctuation smaller than the dimensions of the simulation. Switching dynamics clearly appear to be due to domain wall motion. At higher temperatures, more sporadic nucleation events occur, but they are short-lived and add to noise rather than to signal. At extremely large amplitudes 


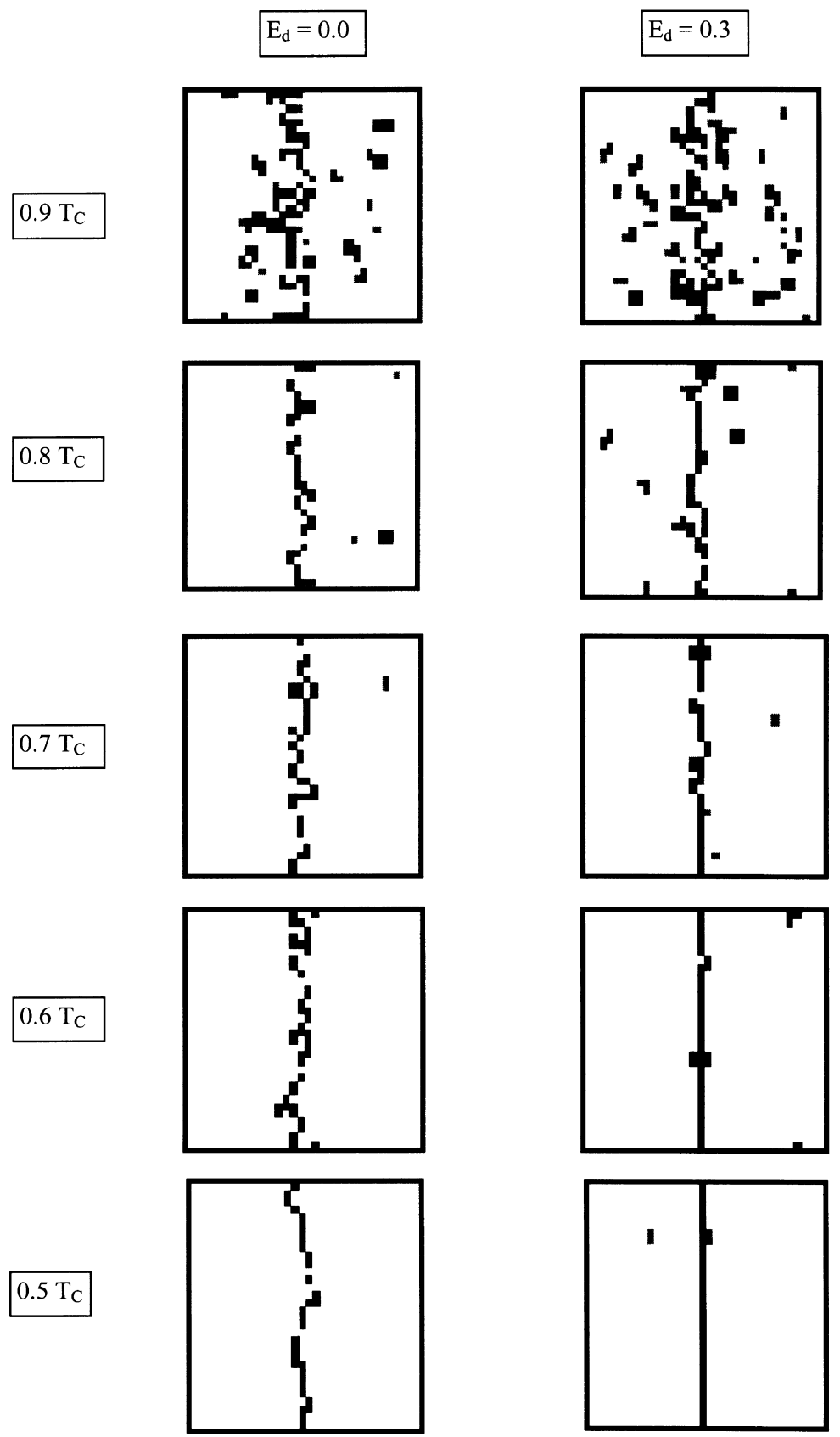

Fig. 4. Domain wall texture as a function of temperature in zero applied field The temperature scale has been adjusted for each case to read as a percent of the transition temperature. The slice images show the Slater and Takagi groups in dark. Both positive and negative polarized groups are white.

of the external fiel (on the order of 0.7 ), an occasional nucleation site will form and grow.

We are interested in the domain wall "sticking" at temperatures of 1.5 and below, for a fiel amplitude of 0.05 . For a given amplitude of the ac field more sticking occurs as the temperature is reduced until there is no response and the hysteresis curve becomes essentially flat
This behavior is consistent with Bornarel's observation [5] that the freezing temperature $T_{\mathrm{f}}$ is dependent on the amplitude of the applied field The sticking in polarization is associated with times at which the wall becomes essentially fla (zero entropy). This is consistent with the observation that thicker samples have lower $T_{\mathrm{f}}$-i.e. it is more likely to fin small residual fluctuation in the wall at lower 
temperatures for larger samples. At temperatures below 0.6-0.7, the fiel required to induce polarization response grows to over 0.5 . At this level, nucleation can also be induced in the bulk of the domain.

While this Hamiltonian is admittedly overly simplistic in that elastic strain energies are ignored, along with bulk polarization energies, etc., the additional included Ishibashi interaction does produce a domain wall freezing effect. This asymmetric "next nearest neighbor" interaction both removes the degeneracy in static wall formation and ensures domain walls normal to the $a$ or $b$ axes of the crystal and allows the wall entropy to vanish at finit temperature. It produces very fla domain walls that do not occur without the interaction. As the wall becomes completely fla defects disappear and the wall can no longer respond to external fields The wall defects necessary to ensure polarization response are only a few unit cells in width, so, a wall width of a few nanometers is all that appears necessary. This is not a "roughening" transition in that the wall width does not diverge. This does not seem to contradict the observations that the domain walls are fla on a scale comparable with the wavelength of light.

\section{Summary}

We have made a preliminary investigation of the possibility that the domain wall freezing observed in KDP can be explained in terms of a local Ishibashi interaction between protons in nearby hydrogen bonds. This interaction was firs proposed to account for the observation of only certain proton ordering arrangements - antiferroelectric as well as ferroelectric - in KDP-type crystals. Its presence would also drive domain walls to adopt a planar configuration as observed, at low temperatures. An approximate Slater-type argument allows us to relate the size of this interaction to the temperature at which the domain wall changes from narrow and smooth, to wide and textured, upon heating. MC simulations illustrate that the Ishibashi interaction has a strong influenc on the geometry of the model domain wall in the absence of an electric field and upon the mobility of the wall when a fiel is applied.

\section{Acknowledgements}

This work was supported by NSF Grant DMR-9805272.

\section{References}

[1] G. Busch, E. Ganz, Helv. Phys. Acta (1942) 501.

[2] C.C. Stephenson, J.G. Hooley, J. Am. Chem. Soc. 66 (1944) 1397.

[3] B. Zwicker, P. Scherrer, Helv. Phys. Acta 17 (1944) 346.

[4] H.M. Barkla, D.M. Finlayson, Phil. Mag. 44 (1953) 109.

[5] J. Bornarel, J. Appl. Phys. 43 (1972) 3.

[6] J. Bornarel, B. Torche, Ferroelectrics 132 (1992) 271.

[7] Y.N. Huang, X. Li, Y. Ding, Y.N. Wang, H.M. Shen, Z.F. Zhang, C.S. Fang, S.H. Zhuo, P.C.W. Fung, Phys. Rev. B 55 (1997) 16159.

[8] Y. Ishibashi, S. Ohya, Y. Takagi, J. Phys. Soc. Jpn 33 (1972) 1545.

[9] V.H. Schmidt, Proceedings of the 5th Williamsburg Workshop on First Principles Calculations, AIP Conf. Proc. 436 (1998) 192.

[10] A. Sinitski, V.H. Schmidt, Phys. Rev. B 54 (1996) 842. 